\title{
Combined GnRH-agonist and human chorionic gonadotropin trigger improves ICSI cycle outcomes in patients with history of poor fertilization
}

\author{
Rony T. Elias ${ }^{1} \cdot$ Nigel Pereira $^{1} \cdot$ Lisa Artusa $^{1} \cdot$ Amelia G. Kelly $^{1} \cdot$ Monica Pasternak $^{1}$. \\ Jovana P. Lekovich ${ }^{1}$ • Gianpiero D. Palermo ${ }^{1} \cdot$ Zev Rosenwaks $^{1}$
}

Received: 21 February 2017 / Accepted: 29 March 2017 / Published online: 13 April 2017

(C) Springer Science+Business Media New York 2017

\begin{abstract}
Purpose The purpose of this study was to investigate the utility of a combined GnRH-agonist (GnRH-a) and human chorionic gonadotropin (hCG) trigger in improving ICSI cycle outcomes in patients with poor fertilization history after standard hCG trigger in prior ICSI cycles.

Methods Retrospective cohort study. Patients with a fertilization rate of $<20 \%$ in at least two prior ICSI cycles who subsequently underwent another ICSI cycle with hCG trigger were compared to those who underwent another ICSI cycle with a combined GnRH-a and hCG trigger. Oocyte maturity, fertilization, clinical pregnancy, and live birth rates were compared. A multiple linear regression model was used to explore the association between combined GnRH-a and hCG trigger (vs hCG trigger alone) and fertilization rate.

Results A total of 427 patients with mean age of $37.3 \pm 1.94$ years and mean baseline fertilization rate of $17.9 \pm 2.03 \%$ were included, of which 318 (74.5\%) and 109 (25.5\%) patients underwent a subsequent ICSI cycle with hCG and combined GnRH-a and hCG trigger, respectively. The baseline parameters of the male
\end{abstract}

Authors R.T.E. and N.P. have contributed equally to this work.

Abstract presented at the American Society of Reproductive Medicine 2016 Scientific Congress and Expo, Salt Lake City, UT, October 15th to 19th 2016

Electronic supplementary material The online version of this article (doi:10.1007/s10815-017-0917-3) contains supplementary material, which is available to authorized users.

Rony T. Elias

rta9002@med.cornell.edu

1 The Ronald O. Perelman and Claudia Cohen Center for Reproductive Medicine, Weill Cornell Medicine, 1305 York Ave, New York, NY 10021, USA and female partner were similar. The mean fertilization rate in the combined trigger group was $16.4 \%$ (95\% CI: $7.58-25.2 \%$ ) higher than the hCG trigger group, even after adjustment for confounders. Patients in the combined trigger group had higher oocyte maturity ( 82.1 vs $69.8 \%$ ), higher clinical pregnancy ( 27.5 vs $5.67 \%$ ), and higher live birth rates (20.2 vs $3.46 \%$ ) compared to the hCG trigger group.

Conclusions Combined GnRH-a and hCG trigger in ICSI cycles increase oocyte maturity, fertilization, clinical pregnancy, and live birth rates in patients with a history of poor fertilization after standard hCG trigger alone.

Keywords Intracytoplasmic sperm injection · Poor fertilization · GnRH-agonist trigger · Dual trigger · ICSI outcomes

\section{Introduction}

The utility of a single bolus of gonadotropin-releasing hormone agonist (GnRH-a) as an alternative to human chorionic gonadotropin (hCG) for triggering final oocyte maturation was first described in 1990 [1]. Since then, several studies have indicated that $\mathrm{GnRH}$-a-based triggers cannot only induce oocyte maturation but can also reduce the risk of ovarian hyperstimulation syndrome in in vitro fertilization (IVF) and intracytoplasmic sperm injection (ICSI) cycles [2-6]. The reduced risk of OHSS has been observed in high or hyperresponders when $\mathrm{GnRH}-$ a triggers are given as a single bolus or in conjunction with low doses of hCG [7-10].

Recent studies have notably highlighted several non-OHSS benefits of pure GnRH-a triggers or combined GnRH-a and hCG triggers in IVF and ICSI cycles [11]. For example, larger cohorts of metaphase II (M-II) oocytes have been obtained in oocyte donors [12], patients with breast cancer [13, 14], and 
patients undergoing IVF/ICSI $[15,16]$ who received GnRH-a triggers compared to standard hCG triggers. The large cohort of M-II oocytes associated with pure or combined GnRH-a triggers in turn increases the chances of shared donor oocyterecipient cycles, efficiency of oocyte banking or oocyte cryopreservation for fertility preservation, as well as supernumerary embryos available for cryopreservation [11]. In some cases, combined GnRH-a and hCG triggers have shown to increase blastulation rates [17] and live birth rates in IVF cycles [9]. Most of these findings have been attributed to the GnRH-a-mediated endogenous surge of follicle-stimulating hormone (FSH) and luteinizing hormone (LH), which resembles the physiologic mid-cycle surge in normal menstrual cycles $[4,18]$.

It is important to note that while the non-OHSS benefits of GnRH-a triggers have only been demonstrated in high or hyper-responders undergoing their first IVF or ICSI cycle, very few studies to date have explored the efficacy of GnRH-a triggers in normal responders or in clinical scenarios such as poor fertilization or unsuccessful IVF/ICSI cycles. Although Griffin et al. [15] have reported increased fertilization rates in 27 patients receiving a combined GnRH-a and hCG trigger (83.3\%) compared to those receiving a standard hCG trigger $(66.7 \%)$, these results were not statistically significant. Also, in a recent investigation, Pereira et al. [19] reported a 2.7 times higher odds of fertilization when normal responder patients with a fertilization rate $<40 \%$ in a prior ICSI cycle with standard hCG trigger underwent a subsequent ICSI cycle with a combined $2 \mathrm{mg} \mathrm{GnRH}-\mathrm{a}$ and $1500 \mathrm{IU}$ hCG ovulatory trigger. However, low fertilization in the prior study was defined by an arbitrary cut-off of $<40 \%$. Given the shortcomings of the aforementioned studies, the primary objective of the current study is to investigate the utility of a combined GnRH-a and hCG ovulatory trigger in improving ICSI cycle outcomes in patients with poor fertilization history following standard hCG trigger in ICSI cycles.

\section{Materials and methods}

\section{Inclusion and exclusion criteria}

All couples presenting for ICSI with fresh day 3 embryo transfer (ET) for male factor infertility at our center during an 8year period were assessed for potential inclusion. From this initial cohort, patients with $>5$ mature oocytes and poor fertilization, defined by a fertilization rate of $<20 \%$, in at least two prior ICSI cycles at our center or elsewhere were included. The cut-off for fertilization rate was decided a priori and represented two standard deviations below the mean fertilization rate at our center $(78.8 \pm 28.5 \%)$. Only normal responders meeting the following criteria were included in the current study: age $<40$ years, cycle day $2 / 3 \mathrm{FSH}$ level $<12 \mathrm{mIU} / \mathrm{mL}$, and cycle day $2 / 3$ anti-müllerian hormone $(\mathrm{AMH})$ level $>1 \mathrm{ng} /$ $\mathrm{mL}$. Patients utilizing donor oocytes, donor sperm, or surgical retrieved sperm were excluded. Based on Pereira et al.'s prior experience of higher fertilization rates with the combined GnRH-a and hCG trigger [19], patients with a fertilization rate of $<20 \%$ in at least two prior successive ICSI cycles prospectively underwent another ICSI cycle with hCG trigger (control group) or with a combined GnRH-a and hCG trigger (study group); the outcomes of these patients were subsequently compared. The decision to proceed with either trigger type was based on physician preference. Our study was approved by the Institutional Review Board.

\section{Clinical, laboratory, and sperm preparation protocols}

All patients underwent uterine cavity evaluation in the form of saline infusion sonography, hysterosalpingography, or hysteroscopy prior to ovarian stimulation. Ovarian stimulation was performed using gonadotropins (Gonal-F, EMD-Serono Inc., Rockland, MA, USA or Follistim, Merck, Kenilworth, NJ, USA; and, Menopur, Ferring Pharmaceuticals Inc., Parsippany, NJ, USA). Dosing of gonadotropins was based on age, body mass index (BMI, $\left.\mathrm{kg} / \mathrm{m}^{2}\right)$, antral follicle count, $\mathrm{AMH}$ level, and response to ovarian stimulation in prior ICSI cycles [20]. All ICSI cycles included in the current study utilized gonadotropin-releasing hormone-antagonist (GnRH-ant) protocols in which suppression of ovulation was achieved with daily injections of ganirelix acetate (Merck, Kenilworth, NJ, USA) based on a previously described flexible protocol [21]. All patients in the hCG trigger group received 10,000 IU hCG while patients in the combined GnRHa and hCG trigger group received $4 \mathrm{mg} \mathrm{GnRH-a}$ and $10,000 \mathrm{IU}$ hCG when the two lead follicles attained a mean diameter $>17 \mathrm{~mm}$. Oocyte retrievals were performed approximately $34-35 \mathrm{~h}$ after the ovulatory trigger under sedation with transvaginal ultrasound guidance. All patients received intramuscular progesterone (50 mg daily) for luteal support.

The retrieved oocytes were exposed to $40 \mathrm{IU}$ recombinant hyaluronidase (Cumulase, Halozyme Therapeutics, Inc. San Diego, CA, USA) to remove the cumulus cells [22]. Metaphase-II (M-II) oocytes were identified by the presence of the first polar body [23]. Selection, immobilization, and permeabilization of the spermatozoa, with subsequent microinjection of M-II oocytes, were performed according to previously described protocols [24]. Of note, semen samples of the male partner were evaluated for volume, count, concentration, and motility using WHO criteria [25] at the first consultation as well as on the on the day of oocyte retrieval. All injected MII oocytes were examined approximately $14-18 \mathrm{~h}$ after ICSI for normal fertilization, i.e., the presence of two distinct pronuclei (2-PN) and two clear polar bodies [22, 23]. The resulting embryos were cultured in in-house culture media until cleavage stage [24]. Fresh ETs were performed with 
Wallace catheters (Smiths Medical Inc., Norwell, MA, USA) on day 3 approximately $1 \mathrm{~cm}$ less than the uterine depth identified at prior trial transfer. There were no major changes in laboratory conditions, culture media, or fresh ET technique during the study period. By study design, patients undergoing embryo biopsy for pre-implantation genetic diagnosis or screening were excluded.

\section{Study variables}

Baseline demographics recorded for each patient included age, BMI $\left(\mathrm{kg} / \mathrm{m}^{2}\right)$, gravidity, cycle day $2 / 3$ folliclestimulating hormone (FSH, $\mathrm{mIU} / \mathrm{mL}$ ) level, and cycle day $2 /$ 3 AMH (ng/mL) level. For the male partner, mean spermatozoa count $\left(\times 10^{6} / \mathrm{mL}\right)$, spermatozoa motility (\%), and morphology $(\%)$ were also recorded. Baseline ICSI cycle characteristics recorded were number of prior ICSI cycles, fertilization rate in prior ICSI cycles, and mean 2-PN embryos obtained in prior ICSI cycles. The following ovarian stimulation parameters were also recorded: total days of ovarian stimulation, total days of gonadotropin-releasing hormone-antagonist (GnRHant) administration, total dosage of gonadotropins administered (IU), peak endometrial thickness ( $\mathrm{mm}$ ), and total and mature oocytes retrieved. The time elapsed between trigger and oocyte retrieval, trigger and removal of cumulus cells, and trigger and sperm injection was also recorded. Hormonal measurements included estradiol $\left(\mathrm{E}_{2}, \mathrm{pg} / \mathrm{mL}\right), \mathrm{LH}$ $(\mathrm{mIU} / \mathrm{mL})$, and FSH $(\mathrm{mIU} / \mathrm{mL})$ levels on day of ICSI start, day of trigger, and day after trigger. ICSI outcomes analyzed were fertilization rate (\%), supernumerary embryos available for cryopreservation, number of day 3 embryos transferred, implantation rate, clinical pregnancy rate, and live birth rate. The fertilization rate was defined as the number of 2-PN embryos out of the total number of mature MII oocytes injected. Implantation rate was defined as the mean number of sacs seen on ultrasonography divided by the number of embryos transferred for each patient undergoing ET. Clinical pregnancy rate was defined as the number of intrauterine gestations with fetal cardiac activity per ICSI-ET cycle. Any birth after 24 weeks of gestational age was considered a live birth.

\section{Statistical analyses}

Descriptive statistics (including mean, standard deviation, median, interquartile range [IQR], frequency, and percent) were calculated to characterize the study cohort (for both the control and study groups, separately). To compare continuous variables of interest between the control and study groups, the two sample $t$ test or nonparametric Wilcoxon rank-sum test was used, as appropriate. To compare categorical variables of interest between the control and study groups, the Chi-square test or Fisher's exact test was used, as appropriate. Fertilization rate was considered the primary outcome of interest. A multiple linear regression model was used to assess the independent effect of group status (i.e., combined GnRH-a and hCG trigger vs hCG trigger alone) on the fertilization rate, after controlling for age, total days of ovarian stimulation, total days of GnRH-ant administration, total dosage of gonadotropins administered, and $\mathrm{E}_{2}$ level on the trigger. Statistical significance was set at $P<0.05$. Ninety-five percent confidence intervals $(95 \% \mathrm{CI})$ were calculated to assess the precision of the obtained estimates. All statistical analyses were performed using STATA version 14.0 (College Station, TX: StataCorp LP).

\section{Results}

A total of 427 patients with $>5$ mature oocytes and $<20 \%$ fertilization rate in at least two prior successive ICSI cycles were identified. These patients had a mean age of $37.3 \pm 1.94$ years and underwent $2.77 \pm 1.40$ prior ICSI cycles, with a mean fertilization rate of $17.9 \pm 2.03 \%$. The mean number of 2-PN embryos was $2.56 \pm 0.63$. The overall positive pregnancy rate in this cohort was $4.21 \%$; however, no live births occurred.

Of the 427 patients, 318 (74.5\%) and 109 (25.5\%) patients underwent a subsequent ICSI cycle with hCG and combined GnRH-a and hCG trigger, respectively. Table 1 compares the baseline demographics and ICSI cycle characteristics of the hCG and combined GnRH-a and hCG trigger groups. No difference was noted in the baseline parameters of the male or female partner in both groups. In addition, both groups underwent a similar number of prior ICSI cycles, had similar mean fertilization rates and a comparable number of 2-PN embryos. Table 2 summarizes the ovarian stimulation parameters of the study cohort. No difference was noted in the total days of ovarian stimulation, total days of GnRH-ant administration, total dosage of gonadotropins administered, or peak endometrial thickness. Although the total number of oocytes was comparable, more M-II oocytes were retrieved in the combined GnRH-a and hCG trigger group [8 (4-11) vs 6 (4-10); $P=0.02]$. There was no difference in the times between trigger and oocyte retrieval, trigger and removal of cumulus cells, and trigger and ICSI when comparing the groups.

As illustrated in Fig. 1a, the $\mathrm{E}_{2}$ levels on the day of ICSI start, day of trigger, and day after trigger in the combined GnRH-a and hCG and hCG trigger groups were comparable. In contrast, Fig. 1b shows that the FSH levels on the day after trigger were higher in the combined trigger group compared to levels observed in the hCG trigger group $(45.3 \pm 9.37$ vs $13.3 \pm 1.26 \mathrm{mIU} / \mathrm{mL}$, respectively, $P<0.001$ ). The doubling of FSH levels between the day of and day after trigger in the combined trigger group signifies the GnRH-a induced surge of FSH. Supplemental Fig. 1 indicates the concomitant GnRH-a induced surge of LH in the combined trigger group. 
Table 1 Baseline demographics and ICSI cycle characteristics of study cohort $(n=427)$

\begin{tabular}{llll}
\hline Parameter & $\begin{array}{l}\text { hCG trigger } \\
(n=318)\end{array}$ & $\begin{array}{l}\text { GnRH-a and hCG } \\
\text { trigger }(n=109)\end{array}$ & $P$ \\
\hline Female partner & & & \\
Age (years) & $37.1( \pm 2.21)$ & $37.4( \pm 2.17)$ & 0.22 \\
BMI $\left(\mathrm{kg} / \mathrm{m}^{2}\right)$ & $24.0( \pm 0.55)$ & $24.2( \pm 0.46)$ & 0.33 \\
Gravidity & $1.39( \pm 0.72)$ & $1.47( \pm 0.68)$ & 0.31 \\
Cycle day 2/3 FSH $(\mathrm{mIU} / \mathrm{mL})$ & $4.48( \pm 3.75)$ & $4.19( \pm 2.42)$ & 0.45 \\
Cycle day 2/3 AMH $(\mathrm{ng} / \mathrm{mL})$ & $1.19( \pm 0.22)$ & $1.21( \pm 0.25)$ & 0.43 \\
Male partner & & & 0.31 \\
Sperm count $\left(\times 10^{6} / \mathrm{mL}\right)$ & $10.8( \pm 3.71)$ & $11.2( \pm 2.91)$ & 0.19 \\
Sperm motility $(\%)$ & $19.8( \pm 9.26)$ & $21.1( \pm 7.72)$ & 0.99 \\
Sperm morphology $(\%)$ & $1(0-2)$ & $1(0-2)$ & 0.10 \\
Couple & & & 0.14 \\
Number of prior ICSI cycles & $2.75( \pm 0.71)$ & $2.88( \pm 0.69)$ & $2.58( \pm 0.67)$ \\
2-PN embryos obtained in prior ICSI cycles & $2.49( \pm 0.51)$ & & \\
\hline
\end{tabular}

Data are presented as mean \pm standard deviation

$B M I$ body mass index, FSH follicle-stimulating hormone, AMH anti-Müllerian hormone, ICSI intracytoplasmic sperm injection, 2-PN 2-pronuclei
Figure 2a demonstrates that the mean fertilization rate in the combined GnRH-a and hCG group was significantly higher than the hCG trigger group $(42.1 \pm 10.8$ vs $17.9 \pm 3.61 \%$, respectively, $P<0.001)$. Multiple linear regression analysis revealed the mean fertilization rate in the combined trigger group to be $16.4 \%$ (95\% CI: $7.58-25.2 \%$ ) higher than the hCG trigger group, after controlling for age, total days of ovarian stimulation, total days of GnRH-ant administration, total dosage of gonadotropins administered, and $\mathrm{E}_{2}$ level on the day of trigger (Table 3).

A significantly higher percentage of ICSI cycles resulted in ET in the combined trigger group (76.1\%) compared to the hCG trigger group $(22.1 \% ; P<0.001)$. Although there was an observed statistical difference in the supernumerary embryos available for cryopreservation when comparing the combined trigger and hCG trigger groups $(0.58 \pm 0.14$ vs $0.31 \pm 0.08$,
Table 2 Ovarian stimulation outcomes of the study cohort $(n=427)$

\begin{tabular}{lllc}
\hline Parameter & $\begin{array}{l}\text { hCG trigger } \\
(n=318)\end{array}$ & $\begin{array}{l}\text { GnRH-a and hCG } \\
\text { trigger }(n=109)\end{array}$ & $P$ \\
\hline Total stimulation days & $10.7( \pm 2.67)$ & $10.8( \pm 3.09)$ & 0.75 \\
Total GnRH-ant days & $5.75( \pm 2.82)$ & $5.49( \pm 2.61)$ & 0.40 \\
Total gonadotropins administered & $2720.9( \pm 753.2)$ & $2741.2( \pm 761.7)$ & 0.36 \\
Peak endometrial thickness (mm) & $10.5( \pm 2.92)$ & $10.6( \pm 2.93)$ & 0.94 \\
Total oocytes retrieved & $9(5-14)$ & $10(5-13)$ & 0.56 \\
Metaphase-II oocytes retrieved & $6(4-10)$ & $8(4-11)$ & 0.02 \\
Metaphase-II oocytes $(\%)$ & $69.8 \%$ & $82.1 \%$ & 0.03 \\
Time between trigger and oocyte retrieval (mins) & $2173.5( \pm 21.4)$ & $2169.3( \pm 25.7)$ & 0.10 \\
Time between trigger and removal of cumulus cells $($ mins $)$ & $2235.4( \pm 28.7)$ & $2240.1( \pm 27.1)$ & 0.14 \\
Time between trigger and ICSI (mins) & $2381.3( \pm 19.7)$ & $2379.0( \pm 20.5)$ & 0.30 \\
Day 3 embryos transferred & $2.51( \pm 0.89)$ & $2.67( \pm 0.64)$ & 0.08 \\
Implantation rate $(\%)$ & $10.7 \%$ & $18.1 \%$ & 0.13 \\
Clinical pregnancy rate $(\%)$ & $18(5.67 \%)$ & $30(27.5 \%)$ & $<0.001$ \\
Live birth rate $(\%)$ & $11(3.46 \%)$ & $22(20.2 \%)$ & $<0.001$ \\
Twin live birth rate $(\%)$ & $1 / 11(9.1 \%)$ & $3(13.6 \%)$ & 0.74 \\
\hline
\end{tabular}

Data are presented as mean \pm (standard deviation), $n(\%)$, and median (interquartile range)

GnRH-ant gonadotropin releasing hormone-antagonist, ICSI intracytoplasmic sperm injection 
Fig. 1 Comparison of estradiol levels (a) and follicle-stimulating hormone levels (b) at the start of cycle, day of trigger, and day after trigger in patients receiving either standard hCG trigger or combined GnRH-a and hCG trigger

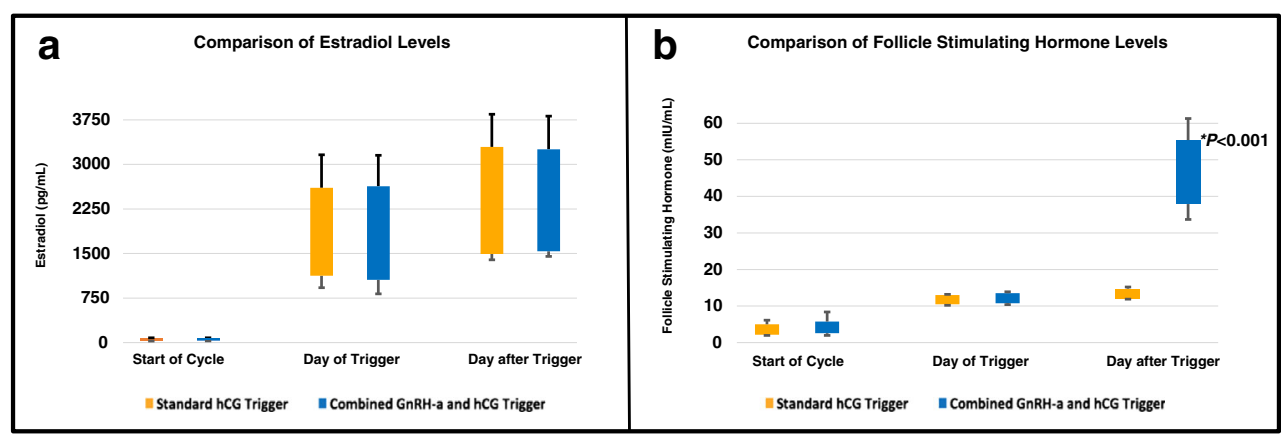

respectively, $P<0.001$ ), the overall number of supernumerary embryos was low. Table 2 shows a similar number of embryos transferred and implantation rate in both trigger groups. Table 2 and and Fig. 2b compare the clinical pregnancy and live birth rates of the two groups. The combined trigger group was noted to have a higher clinical pregnancy rate (27.5 vs $5.67 \%$, respectively, $P<0.001)$ and higher live birth rate ( 20.2 vs $3.46 \%$, respectively, $P<0.001)$ compared to the hCG trigger group.

\section{Discussion}

The results of the current study indicate that normal responder patients with a fertilization rate of $<20 \%$ in at least two prior ICSI cycles for male-factor infertility have higher mean fertilization rates in a subsequent ICSI cycle when utilizing a combined GnRH-a and hCG trigger compared to a standard hCG trigger. The mean fertilization rate in the combined trigger group was approximately $16.4 \%$ higher than the rate in hCG trigger group, even after adjusting for confounders with multiple linear regression analysis. Furthermore, patients in the combined trigger group had more M-II oocytes retrieved, a higher percentage of ICSI cycles resulting in day $3 \mathrm{ET}$, higher clinical pregnancy rates, and higher live birth rates.

Despite the common utilization of ICSI, cases of poor fertilization or complete fertilization failure can occur, often in the same patient [26]. Poor fertilization even in the presence of an adequate number of M-II oocytes and the same sperm source can prove to be frustrating for patients and physicians alike. Previous studies have suggested that endogenous FSH levels have a stimulatory effect on oocyte competence [27] by increasing in vivo granulosa-granulosa and granulosa-oocyte gap junctional communication $[28,29]$. Thus, low endogenous FSH levels due to intentional pituitary suppression during ovarian stimulation may negatively influence the developmental competence of oocytes, resulting in poor ICSI fertilization in some patients with adequate M-II oocytes [27]. A proposed mechanism for poor fertilization in such patients may be asynchrony between the nuclear and ooplasmic maturation of the oocyte $[30,31]$. In other words, in the setting of low endogenous FSH levels, M-II oocytes can contain immature ooplasm even though they are deemed mature for sperm injection by the presence of the first polar body, i.e., nuclear maturity. Such M-II oocytes are unable to participate in the decondensation of the sperm nucleus, leading to poor or failed oocyte fertilization $[32,33]$.

In the context of these findings, investigators have assessed whether a bolus of FSH given concomitantly with the standard hCG trigger may be beneficial in increasing fertilization rates with ICSI. Specifically, Lamb et al. [27] randomized 188 patients undergoing IVF or ICSI to a bolus of 450 IU FSH or placebo at the time of $10,000 \mathrm{IU}$ hCG trigger and reported higher oocyte recovery rates (69.9 vs $57.1 \%$ ) and mean fertilization rates (63 vs 55\%) in the FSH bolus group. Of note, when patients undergoing IVF and ICSI were analyzed separately, statistically higher mean fertilization rates were only noted in the IVF group (68 vs 48\%) receiving the FSH bolus. No difference in clinical pregnancy or live birth rates was reported in that study. Contrary to the increased mean fertilization rates highlighted by Lamb et al. [27], other investigations have failed to demonstrate any improvement in
Fig. 2 Comparison of mean fertilization rates (a), clinical pregnancy rates, and live birth rates (b) in patients receiving either standard hCG trigger or combined GnRH-a and hCG trigger

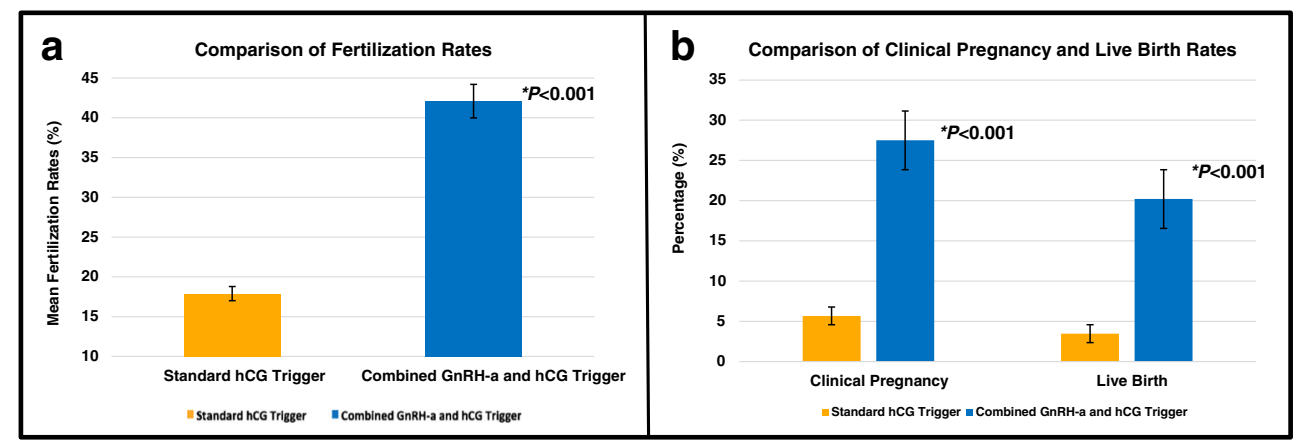


Table 3 Multiple linear regression to explore the association between combined GnRH-a and hCG trigger (vs hCG trigger alone) and fertilization rate

\begin{tabular}{lllll}
\hline Parameter & Coefficient & Standard error & $\begin{array}{l}95 \% \text { confidence } \\
\text { intervals }\end{array}$ & $P$ \\
\hline $\begin{array}{l}\text { Combined GnRH-a and hCG trigger vs hCG } \\
\text { trigger (referent) }\end{array}$ & 16.4 & 4.48 & $7.58,25.2$ & $<0.001$ \\
$\begin{array}{l}\text { Age (years) } \\
\text { Total stimulation days }\end{array}$ & 2.12 & 2.16 & $-2.12,6.36$ & 0.33 \\
Total GnRH-ant days & 0.86 & 2.18 & $-3.42,5.14$ & 0.69 \\
Total gonadotropins administered (IU) & 4.49 & 3.18 & $-1.77,10.7$ & 0.16 \\
E $_{2}$ level on the day of trigger (pg/mL) & -1.97 & 2.62 & $-7.11,3.16$ & 0.45 \\
\hline
\end{tabular}

$G n R H$-ant gonadotropin-releasing hormone-antagonist, $E_{2}$ estradiol fertilization, clinical pregnancy, or live birth rates in patients receiving an FSH bolus with the hCG trigger [34, 35].

It is worthwhile to note that although increased serum FSH levels were observed on the day after trigger in the current and previous studies [27, 34], the mechanism causing increased FSH levels is different - the higher serum FSH levels in the current study are endogenous in origin, resulting from the GnRH-a-mediated surge in FSH, while the higher serum FSH levels in other studies are due to administration of exogenous FSH. Given that higher fertilization, clinical pregnancy, and live birth rates were only noted in the current study, one may speculate that the biologic effects of endogenous FSH on oocyte developmental competence are more potent than exogenous FSH. A recent prospective study by Haas et al. [36] has elucidated some physiologic mechanisms pertaining to the beneficial effects of the GnRH-a-mediated FSH surge in ICSI cycles. In this study, 15 patients received a standard hCG trigger in the first ICSI cycle followed by a combined GnRH-a and hCG trigger in the subsequent ICSI cycle. Granulosa cells were obtained at the time of oocyte retrieval from which RNA was extracted. Expression analysis of messenger RNA (mRNA) levels of reproduction-related genes was then performed with quantitative real-time polymerase chain reaction. Consistent with the findings of the current study, Haas et al. also reported a higher mean number of oocytes (8.6 vs 6.9), 2-PN embryos (6.1 vs 3.7), cleavage-stage embryos (5.6 vs 3.4), and higher clinical pregnancy rates in the combined trigger group compared to that of the hCG trigger group. The mRNA expression of amphiregulin and epiregulin was significantly higher in the combined trigger group. FSH has been shown to increase the expression of amphiregulin and epiregulin in granulosa cells, and these genes in turn induce cumulus expansion and oocyte maturation $[37,38]$. In vitro experiments have also shown that addition of amphiregulin and epiregulin to media can significantly increase the maturation of germinal vesicle oocytes. The investigators [36] also reported significantly lower expression of connexin 43 in the combined trigger group. Previous studies have indicated that embryos with good morphology on day 3 ( $>7$ cells and $<10 \%$ fragmentation) develop from oocytes with lower mRNA expression of connexin 43 [39]. Thus, the surge in endogenous FSH associated with GnRH-a results in higher levels of amphiregulin and epiregulin as well as connexin 43 suppression; these combined mechanisms potentially contribute to increased oocyte maturity, fertilization rates, and live birth rates in patients receiving the combined GnRH-a and hCG trigger.

One salient strength of the current study is the inclusion of patients with $>5$ mature oocytes undergoing ICSI with ejaculated sperm, reducing the possibility of poor fertilization in such patients being caused by low oocyte yield [40] or severe sperm defects [41]. Although the semen parameters of the male partners in the current study were suboptimal, several studies have indicated that ICSI can achieve normal fertilization rates despite suboptimal semen parameters [22, 23]. Furthermore, to address regression to the mean as a potential source of error, only patients with $<20 \%$ fertilization rate in at least two prior ICSI cycles were included. The occurrence of $<20 \%$ fertilization in two or more prior ICSI cycles also indicates a biologic and patient-specific basis for poor fertilization, most likely at the oocyte level [19]. Of note, the cut-off for fertilization rate was decided a priori and was center-specific, i.e., two standard deviations below the center's mean fertilization rate.

The current study also has some limitations. First, only patients undergoing ovarian stimulation with GnRHantagonist protocols, ICSI for male factor infertility, and fresh day 3 ET were included. Thus, by study design, the utility of the combined GnRH-a and hCG trigger could not be assessed in patients undergoing ovarian stimulation with non-GnRHantagonist protocols, ICSI for other infertility diagnoses, or those with blastocyst-stage transfers. Second, information regarding smoking status was not available and therefore, could not be analyzed as a potential confounder. Third, while the study postulated that higher endogenous FSH levels associated with the combined trigger can mitigate asynchrony between the nuclear and ooplasmic maturation of the oocyte, ooplasmic maturity was not specifically assessed in the current study. Non-invasive imaging of the meiotic spindle has previously been utilized to determine the ooplasmic maturity 
of human oocytes. However, there is conflicting evidence regarding the association of the meiotic spindle with outcomes such as fertilization rates, embryo quality, implantation rates, and clinical pregnancy rates [42]. Fourth, we also acknowledge that poor fertilization may be caused by poor quality oocytes generated from sub-optimal ovarian stimulation. Finally, the results of the current retrospective study encourage replication in a prospective setting. It would also be worthwhile to explore whether the pure $\mathrm{GnRH}$-a triggers, i.e., GnRH-a without the hCG component, could have a direct impact on oocyte maturity and fertilization rates.

The current study adds to the growing body of literature demonstrating the non-OHSS benefits of GnRH-a triggers in assisted reproduction. Specifically, a combined GnRH-a and hCG ovulatory trigger is associated with increased mean fertilization rates and increased clinical pregnancy and live birth rates in patients with poor fertilization in prior ICSI cycles with standard hCG trigger alone. The study's findings are particularly important for normal responder patients with recurrent poor fertilization, who otherwise have very limited treatment options [15]. While the study postulates that the GnRH-a-mediated surge in endogenous FSH can mitigate ooplasmic immaturity and thereby increase fertilization and pregnancy rates, basic scientific investigations are required to confirm the proposed aforementioned mechanism.

Acknowledgements We thank Dr. Paul J. Christos for his assistance with statistical analyses and Logan Stone for his editorial assistance.

\section{Compliance with ethical standards}

Conflict of interest The authors declare that they have no conflict of interest.

\section{References}

1. Gonen Y, Balakier H, Powell W, Casper RF. Use of gonadotropinreleasing hormone agonist to trigger follicular maturation for in vitro fertilization. J Clin Endocrinol Metab. 1990;71(4):918-22.

2. Youssef MA, Van der Veen F, Al-Inany HG, Griesinger G, Mochtar $\mathrm{MH}$, Aboulfoutouh I, et al. Gonadotropin-releasing hormone agonist versus HCG for oocyte triggering in antagonist assisted reproductive technology cycles. Cochrane Database Syst Rev. 2011;1: CD008046.

3. Fatemi HM, Garcia-Velasco J. Avoiding ovarian hyperstimulation syndrome with the use of gonadotropin-releasing hormone agonist trigger. Fertil Steril. 2015;103(4):870-3.

4. Humaidan P, Papanikolaou EG, Tarlatzis BC. GnRHa to trigger final oocyte maturation: a time to reconsider. Hum Reprod. 2009;24(10):2389-94.

5. Casper RF. Reducing the risk of OHSS by GnRH agonist triggering. J Clin Endocrinol Metab. 2015;100(12):4396-8.

6. Griesinger G, Diedrich K, Devroey P, Kolibianakis EM. GnRH agonist for triggering final oocyte maturation in the GnRH antagonist ovarian hyperstimulation protocol: a systematic review and meta-analysis. Hum Reprod Update. 2006;12(2):159-68.
7. Shapiro BS, Daneshmand ST, Garner FC, Aguirre M, Hudson C. Comparison of "triggers" using leuprolide acetate alone or in combination with low-dose human chorionic gonadotropin. Fertil Steril. 2011;95(8):2715-7.

8. Engmann L, DiLuigi A, Schmidt D, Nulsen J, Maier D, Benadiva C. The use of gonadotropin-releasing hormone (GnRH) agonist to induce oocyte maturation after cotreatment with GnRH antagonist in high-risk patients undergoing in vitro fertilization prevents the risk of ovarian hyperstimulation syndrome: a prospective randomized controlled study. Fertil Steril. 2008;89(1):84-91.

9. Griffin D, Benadiva C, Kummer N, Budinetz T, Nulsen J, Engmann L. Dual trigger of oocyte maturation with gonadotropin-releasing hormone agonist and low-dose human chorionic gonadotropin to optimize live birth rates in high responders. Fertil Steril. 2012;97: 1316-20.

10. Pabuccu EG, Pabuccu R, Caglar GS, Y1lmaz B, Yarc1 A. Different gonadotropin releasing hormone agonist doses for the final oocyte maturation in high-responder patients undergoing in vitro fertilization/intra-cytoplasmic sperm injection. J Hum Reprod Sci. 2015;8(1):25-9.

11. Shapiro BS, Andersen CY. Major drawbacks and additional benefits of agonist trigger-not ovarian hyperstimulation syndrome related. Fertil Steril. 2015;103(4):874-8.

12. Shapiro BS, Daneshmand ST, Garner FC, Aguirre M, Ross R. Comparison of human chorionic gonadotropin and gonadotropinreleasing hormone agonist for final oocyte maturation in oocyte donor cycles. Fertil Steril. 2007;88(1):237-9.

13. Oktay K, Türkçüoğlu I, Rodriguez-Wallberg KA. GnRH agonist trigger for women with breast cancer undergoing fertility preservation by aromatase inhibitor/FSH stimulation. Reprod BioMed Online. 2010;20(6):783-8.

14. Reddy J, Turan V, Bedoschi G, Moy F, Oktay K. Triggering final oocyte maturation with gonadotropin-releasing hormone agonist (GnRHa) versus human chorionic gonadotropin (hCG) in breast cancer patients undergoing fertility preservation: an extended experience. J Assist Reprod Genet. 2014;31(7):927-32.

15. Griffin D, Feinn R, Engmann L, Nulsen J, Budinetz T, Benadiva C. Dual trigger with gonadotropin-releasing hormone agonist and standard dose human chorionic gonadotropin to improve oocyte maturity rates. Fertil Steril. 2014;102(2):405-9.

16. Humaidan P, Bredkjaer HE, Bungum L, Bungum M, Grøndahl ML, Westergaard L, et al. GnRH agonist (buserelin) or hCG for ovulation induction in GnRH antagonist IVF/ICSI cycles: a prospective randomized study. Hum Reprod. 2005;20(5):1213-20.

17. Werner MD, Forman EJ, Hong KH, Franasiak JM, Neal SA, Scott Jr RT. Dual trigger with GnRH agonist (GnRHa) and varying doses of hCG increases the blastulation rate amongst high responders. Fertil Steril. 2014;102(3):Supplement e220-1.

18. Casper RF. Basic understanding of gonadotropin-releasing hormone-agonist triggering. Fertil Steril. 2015;103(4):867-9.

19. Pereira N, Elias RT, Neri QV, Gerber RS, Lekovich JP, Palermo $\mathrm{GD}$, et al. Adjuvant gonadotrophin-releasing hormone agonist trigger with human chorionic gonadotrophin to enhance ooplasmic maturity. Reprod BioMed Online. 2016;33(5):568-74.

20. Huang JY, Rosenwaks Z. Assisted reproductive techniques. Methods Mol Biol. 2014;1154:171-231.

21. Pereira N, Reichman DE, Goldschlag DE, Lekovich JP, Rosenwaks Z. Impact of elevated peak serum estradiol levels during controlled ovarian hyperstimulation on the birth weight of term singletons from fresh IVF-ET cycles. J Assist Reprod Genet. 2015;32(4): 527-32.

22. Palermo GD, Neri QV, Schlegel PN, Rosenwaks Z. Intracytoplasmic sperm injection (ICSI) in extreme cases of male infertility. PLoS One. 2014;9(12):e113671. 
23. Palermo GD, Cohen J, Alikani M, Adler A, Rosenwaks Z. Intracytoplasmic sperm injection: a novel treatment for all forms of male factor infertility. Fertil Steril. 1995;63(6):1231-40.

24. Gosden LV. Oocyte retrieval and quality evaluation. Methods Mol Biol. 2014;1154:343-60.

25. World Health Organization. WHO laboratory manual for the examination and processing of human semen. 5th ed. Geneva: WHO Press, World Health Organization; 2010.

26. Palermo GD, Kocent J, Monahan D, Neri QV, Rosenwaks Z. Treatment of male infertility. Methods Mol Biol. 2014;1154:385405.

27. Lamb JD, Shen S, McCulloch C, Jalalian L, Cedars MI, Rosen MP. Follicle-stimulating hormone administered at the time of human chorionic gonadotropin trigger improves oocyte developmental competence in in vitro fertilization cycles: a randomized, doubleblind, placebo-controlled trial. Fertil Steril. 2011;95(5):1655-60.

28. El-Hayek S, Clarke HJ. Follicle-stimulating hormone increases gap Junctional communication between somatic and germ-line follicular compartments during murine oogenesis. Biol Reprod. 2015;93(2):47.

29. Carabatsos MJ, Sellitto C, Goodenough DA, Albertini DF. Oocytegranulosa cell heterologous gap junctions are required for the coordination of nuclear and cytoplasmic meiotic competence. Dev Biol. 2000;226(2):167-79.

30. Sundström P, Nilsson BO. Meiotic and cytoplasmic maturation of oocytes collected in stimulated cycles is asynchronous. Hum Reprod. 1988;3(5):613-9.

31. Pereira N, Neri QV, Lekovich JP, Palermo GD, Rosenwaks Z. The role of in-vivo and in-vitro maturation time on ooplasmic dysmaturity. Reprod BioMed Online. 2016;32(4):401-6.

32. Rawe VY, Olmedo SB, Nodar FN, Doncel GD, Acosta AA, Vitullo $\mathrm{AD}$. Cytoskeletal organization defects and abortive activation in human oocytes after IVF and ICSI failure. Mol Hum Reprod. 2000;6:510-6.

33. Hyun CS, Cha JH, Son WY, Yoon SH, Kim KA, Lim JH. Optimal ICSI timing after the first polar body extrusion in in vitro matured human oocytes. Hum Reprod. 2007;22(7):1991-5.
34. Vermeiden JP, Roseboom TJ, Goverde AJ, Suchartwatnachai C, Schoute E, Braat DD, et al. An artificially induced follicle stimulating hormone surge at the time of human chorionic gonadotrophin administration in controlled ovarian stimulation cycles has no effect on cumulus expansion, fertilization rate, embryo quality and implantation rate. Hum Reprod. 1997;12(7):1399-402.

35. Juneau CR, Morin SJ, Franasiak JM, Landis JN, Molinaro TA, Scott RT. A 'follicle-stimulating hormone boost' administered at the time of human chorionic gonadotropin trigger does not affect IVF cycle outcomes. Fertil Steril. 2016;106(3):Supplement e189-90.

36. Haas J, Ophir L, Barzilay E, Machtinger R, Yung Y, Orvieto R, et al. Standard human chorionic gonadotropin versus double trigger for final oocyte maturation results in different granulosa cells gene expressions: a pilot study. Fertil Steril. 2016;106(3):653-9. e1

37. Park JY, Su YQ, Ariga M, Law E, Jin SL, Conti M. EGF-like growth factors as mediators of LH action in the ovulatory follicle. Science. 2004;303(5658):682-4.

38. Ben-Ami I, Komsky A, Bern O, Kasterstein E, Komarovsky D, Ron-El R. In vitro maturation of human germinal vesicle-stage oocytes: role of epidermal growth factor-like growth factors in the culture medium. Hum Reprod. 2011;26:76-81.

39. Hasegawa J, Yanaihara A, Iwasaki S, Mitsukawa K, Negishi M, Okai T. Reduction of connexin 43 in human cumulus cells yields good embryo competence during ICSI. J Assist Reprod Genet. 2007;24(10):463-6.

40. Swain JE, Pool TB. ART failure: oocyte contributions to unsuccessful fertilization. Hum Reprod Update. 2008;14(5):431-46.

41. Esfandiari N, Javed MH, Gotlieb L, Casper RF. Complete failed fertilization after intracytoplasmic sperm injectionanalysis of 10 years' data. Int J Fertil Womens Med. 2005;50(4):187-92.

42. Petersen CG, Oliveira JB, Mauri AL, Massaro FC, Baruffi RL, Pontes A, et al. Relationship between visualization of meiotic spindle in human oocytes and ICSI outcomes: a meta-analysis. Reprod BioMed Online. 2009;18(2):235-43. 